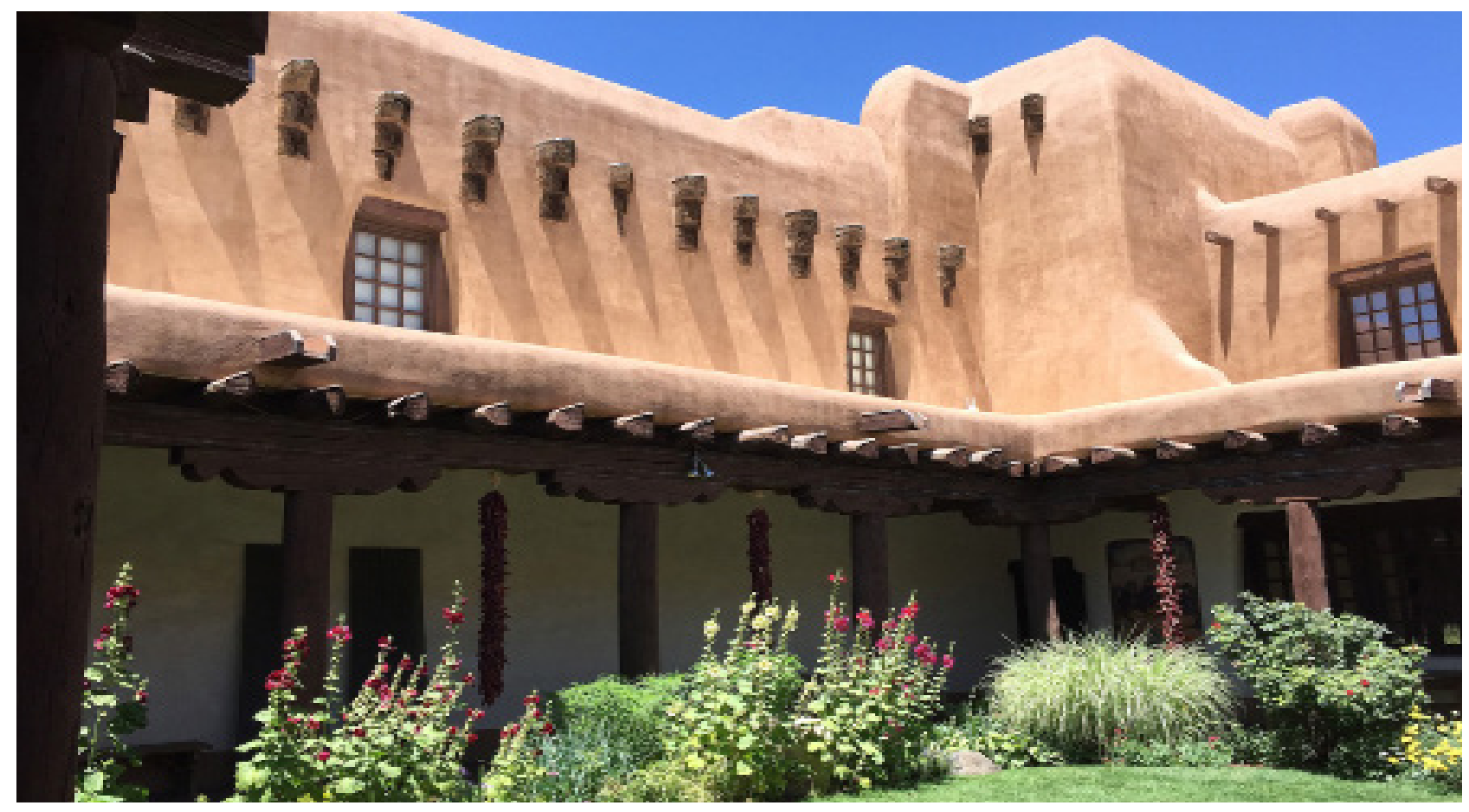

\title{
Usos del adobe en diferentes países de América Latina
}

\section{Uses of adobe in different Latin American countries}

María Gabriela Díaz-Torres

Estudiante Ingeniería Civil, mariagabrieladt@ufps.edu.co,

https://orcid.org/0000-0002-6355-7725, Universidad Francisco de Paula

Santander, Cúcuta, Colombia

Hellen Paola Parada-Carrillo

Estudiante Ingeniería Civil, hellenpaolapc@ufps.edu.co

https://orcid.org/0000-0001-5863-6114, Universidad Francisco de Paula

Santander, Cúcuta, Colombia

María José Alvarado-Arias

Estudiante Ingeniería Civil, mariajoseaa@ufps.edu.co

https://orcid.org/0000-0002-8699-3204, Universidad Francisco de Paula

Santander, Cúcuta, Colombia 


\section{Resumen}

El presente artículo presenta los resultados del análisis comparativo del uso del adobe en diferentes países latinoamericanos que son Perú, México y Colombia. En este análisis se observó el uso que le dan en cada uno de estos países, los métodos constructivos usados y se analizó las construcciones hechas en adobe ya realizadas en diferentes lugares, también se observaron investigaciones llevadas a cabo en estos países basadas en el tema del adobe, analizando el contenido de cada una de estas y las conclusiones que se obtuvieron. Esta investigación se llevó a cabo a partir de un estudio cualitativo, recolectando información a partir de diferentes fuentes bibliográficas, con esta investigación se abordó el tema del adobe que ha sido utilizado por miles de años en diferentes regiones del mundo, se planteó como objetivo principal, el analizar y comparar con base a recopilaciones de otras investigaciones basadas en el adobe de países latinoamericanos como los ya nombrados, pero dando énfasis a la implementación en los procesos constructivos en Colombia, ventajas y beneficios que tiene la utilización de este material en las edificaciones. Como conclusiones podemos observar la inclusión nuevamente del uso del adobe en las construcciones tradicionales usadas actualmente, buscando un beneficio tanto ambiental como económico, ayudando a diferentes sectores de la población.

Palabras clave: Adobe, medio ambiente, métodos constructivos, beneficios, análisis.

\section{Abstract}

This article presents the results of the comparative analysis of the use of adobe in different Latin American countries that are Peru, Mexico and Colombia. In this analysis, the use made in each of these countries was observed, the construction methods used, and the constructions made in adobe already carried out in different places were analyzed. Research carried out in these countries based on the theme of construction was also observed. adobe, analyzing the content of each of these and the conclusions that were obtained. This research was carried out based on a qualitative study, collecting information from different bibliographic sources. With this research, the topic of adobe, which has been used for thousands of years in different regions of the world, was addressed as the main objective. , analyze and compare based on compilations of other adobebased research from Latin American countries such as those already mentioned, but emphasizing the implementation of the construction processes in Colombia, advantages and benefits of using this material in buildings. As conclusions we can observe the inclusion again of the use of adobe in the traditional buildings currently used, seeking an environmental and economic benefit, helping different sectors of the population.

Keywords: Adobe, environment, constructive methods, benefits, analysis. 


\section{Introducción}

Desde tiempos inmemorables la tierra ha sido un material relevante para el avance de la humanidad en todos los aspectos, siendo un recurso indispensable para la innovación de la sociedad y por tanto, objeto de estudio. Al pasar el tiempo el hombre ha desarrollado diferentes sistemas constructivos, variando con respecto a su ubicación geográfica, recursos a su alcance y sus necesidades [1].

Hoy en día, los materiales más utilizados para la construcción es el cemento, ladrillos, morteros, hormigón, entre otros; pero estos materiales no contribuyen en el medio ambiente, ya que las infraestructuras hechas de este material una vez acaban su vida útil pasan a ser escombros y no sirven para volver a ser parte de la tierra.

En Latinoamérica la arquitectura de tierra es de gran importancia por su desarrollo específico, por el lugar que ocupan y lo que representan dentro del país en los centros históricos, la arquitectura monumental construida con esta técnica [2]. Por lo tanto el uso de piezas de construcción provenientes del suelo hace de sí una construcción más eficiente.

Así mismo, en las construcciones se puede trabajar con adobe hecha de arcilla y arena, muchas veces mezclada con paja y estiércol para darle mayor cohesión, moldeada en forma de ladrillos, variando su volumen dependiendo del tipo de estructura y del lugar donde se va a utilizar y con esto formar paredes, muros 0 arcos. Los materiales disponibles y las formas de expresión de las distintas culturas generaron diversas técnicas constructivas que emplearon tierra con exclusividad o en combinación con otros materiales de procedencia animal, vegetal y mineral [3].

Las técnicas de construcción en conjunto con los materiales varían dependiendo de ciertas características. Las costumbres constructivas tradicionales, han evolucionado adoptando excelentes apropiaciones locales que en conjunto son componentes importantes en el desarrollo de la arquitectura regional [4] Todos los estudios realizados a los materiales que componen el adobe (arcilla, arena, paja y estiércol) hacen que sea un recurso más seguro y factible a la hora de emprender nuevos proyectos.

Estas costumbres constructivas en Latinoamérica introdujeron nuevas formas de construcción con tierra. En varios pueblos ya existía, en cierta medida, una utilización de estos materiales. En distintos lugares de México, Perú y Colombia las construcciones de adobe son patrimonio para la comunidad, ya que se conserva como tradición [5].

Varios exploradores y colonizadores europeos trajeron nuevas formas de construcción implementando estos materiales. Así mismo, la revolución industrial en esta parte del mundo favoreció a los materiales más modernos y tienden a orientar los materiales de tierra como pobreza. El pasto seco en combinación con el barro permite una adecuada unión, dando resistencia en el medio y evitando que los bloques tengan grietas, además permiten que estos se adhieran adecuadamente para la construcción de muros [6] El aumento de las necesidades y la contaminación en el medio ambiente se debe realizar una revaluación de estas políticas ya planteadas.

Como se menciona anteriormente en Colombia también son utilizados métodos y materiales distintos de construcción pero guardando entre sí una característica importante, el del uso de materiales tradicionales. De acuerdo con las condiciones locales las técnicas de construcción con tierra en Colombia a evolucionado y ha dado lugar a una expresión particular y especial de la arquitectura [7]. Si bien es cierto, la utilización de este tipo de 


\section{6}

materiales trae grandes ventajas, sirve de aislante sonoro y térmico, la restitución de los materiales al suelo, la economía y el alcance. El adobe se encuentra predominante en los altiplanos al igual que la tapia pisada y en la zona indígena de influencia de los guámbianos, el adobe no lleva fibras vegetales [8] Siendo esto una motivación a retomar estos materiales como base primaria para construcciones y debido a sus bajos costo en la adquisición y que no se necesita un personal especializado para la aplicación del mismo.

Por lo tanto, el adobe es una excelente opción para material de construcción, teniendo un fácil acceso y producción del mismo haciéndolo atractivo en varios lugares de la región. Un ejemplo de ello es el municipio de Villa de Leyva, que se dedica a la fabricación y venta de adobes, usando además dentro de la mezcla otros materiales como tamo de trigo, residuos de obra seleccionados y gavera de madera. [9], además autores como [10] señalan el bambú para posible uso en este campo de la construcción, debido a sus bajos valores de combustión que hace menores los riesgos de quema. Es por eso que se debe tener en cuenta las características del material y la determinación de los lugares en que se pueden vivenciar estructuras hechas en adobe y reconocer el desarrollo en los diferentes ámbitos para la producción y distribución del mismo. En los altiplanos cundiboyacense, payanense y nariñense el adobe es parte fundamental de la cultura arquitectónica colombiana en todos los aspectos de la construcción.

En definitiva, la utilización del adobe en distintos lugares va tomando gran relevancia por sus condiciones y beneficios, es por eso, que se comparara las distintas utilizaciones en Colombia, México y Perú, para determinar sus beneficios y las ventajas de la utilización en las construcciones actuales, determinar en qué lugares y bajo qué condiciones físicas y ambientales se puede construir con adobe.
Los procesos constructivos comunes tienen un impacto negativo hacia el medio ambiente, debido los materiales usados y a los procesos utilizados. Por ello podemos ver que el adobe es un material de la región, también empleado en la construcción, principalmente en muros de viviendas, y su fabricación es amigable con el medio ambiente. [11].

A parte de lo anterior, el presente trabajo de investigación tiene como objetivo analizar y comparar con base a recopilaciones de otras investigaciones sobre el adobe realizadas en otros países latinoamericanos, más específicamente en Perú, México y Colombia, esto enfatizando en la implementación en los procesos constructivos, ventajas y beneficios que conlleva el uso de este material. Para cumplir el objetivo principal, se comparará las formas de uso, los métodos constructivos y las construcciones hechas en estos países ya mencionados (Perú, México y Colombia).

Para terminar, se puede observar que el sector de construcción, está cambiando sus métodos constructivos tradicionales, para volver a utilizar la construcción con adobe, ya que estas edificaciones son más armónicas con el medio ambiente, además que como son materiales que se encuentran en nuestra región, eso le permite a las empresas constructoras brindar a los usuarios viviendas más económicas y accesibles, ya que permite una reducción en los costos de obra.

\section{Fundamentación teórica}

A nivel nacional se encontró una investigación de González, la cual es titulada estado actual de la investigación en arquitectura hecha con tierra en Colombia., realizada en el año 2013, dicha investigación tuvo como objetivo nuevas tendencias contemporáneas ambientales asociadas al ahorro energético en la construcción, así mismo se enfocó en una metodología cualitativa en donde obtuvo que la arquitectura hecho con tierra 
retoma valor entre arquitectos y constructores ecológicos, a pesar de ser parte de la historia de nuestras civilizaciones durante miles de años. Actualmente y desde hace unos cuarenta años, se aborda desde diferentes perspectivas a nivel local y mundial. La construcción ecológica de casas en adobe ha vuelto a tomar auge con la diferencia de que, en su versión más profesional, se trata de una técnica de construcción de casas de adobe reforzado, lo cual le da ese deseado carácter antisísmico.

Además, otra investigación encontrada es la de [12] que esta enfocada en la tecnología y construcción en tierra que nos dice lo siguiente: La tierra en la construcción tiene uso desde las primeras construcciones hechas por el hombre. Los materiales disponibles en la zona y las formas como los usaban las distintas culturas crearon diferentes técnicas constructivas cuyo material principal es la tierra y lo combinaron con otros materiales de otras procedencias como la animal, vegetal y mineral [13]. De estos materiales mencionados anteriormente que pueden ser de procedencia animal, vegetal o mineral, algunos aún continúan siendo usados. De la variedad de materiales que se ha dado, las cuales continúan su mayoría en vigencia, se pueden clasificar en general, para estudiar sus características, ventajas y desventajas. Por eso se han dividido en tres grandes grupos: Tierra apisonada, Tierra con entramado, Albañilería [14].

Por otra parte [15].nos da los siguientes resultados de su investigación titulada en "Construccionesdemurosen TapiayBahareque" que da objeto a las nuevas tecnologías de los sistemas de construcción, en donde se han presentado diferentes y variadas alternativas de solución a la problemática del hábitat del hombre moderno, en muchas ocasiones, sin tener en cuenta el rico e inmenso bagaje cultural de las construcciones ya existentes. Los centros históricos de las ciudades, están ahí con su aporte silencioso e involuntario frente al mundo actual; de estos dos mundos existentes y competidores se han tomado parcialmente los enfoques de las tecnologías con las tradiciones y las técnicas apropiadas de cada uno de ellos, lo que ha llevado hoy a idear múltiples frentes en todas las áreas constructivas, involucrando a la ingeniería y la arquitectura, por ello es fundamental el análisis de resultados y sus metodologías anexadas en documentos para el estudio en general del adobe.

Del mismo modo, otra investigación es la de Rivera Torres publicada en el año 2012. Esta denominada como El adobe y otros materiales de sistemas constructivos en tierra cruda: caracterización con fines estructurales en la cual se habla sobre el sistema constructivo utilizado en el adobe. El sistema constructivo en tierra cruda, también llamado como muros en adobe, está formado básicamente por unidades de tierra cruda secadas al sol (adobes) aglutinados con barro, que hace de mortero de pega. Estas unidades son fabricadas de diferentes formas y con composiciones granulométricas diferentes. [16].El adobe es un material muy usado en las construcciones más antiguas, ya que hoy en día por lo general es común ver el uso de ladrillos y concreto, sin embargo aún hoy vemos este tipo de construcciones en adobe que comúnmente se mezclan con otros tipos de materiales y mampostería Es común que en los sistemas constructivos de las construcciones en tierra se presentan mezclas de diferentes tipos de mampostería: mampuestos en sillares de piedra y/o tapiales, y/o muros en adobe. El estudio y conocimiento de las especificaciones de los materiales, de los sistemas constructivos y de la aplicación de estos en las soluciones para rehabilitar los bienes de interés cultural o con carácter histórico, se está realizando en Colombia pero solamente está basado en inspecciones visuales y estudios cualitativos, sin profundizar en estudios cuantitativos que nos permiten tener un conocimiento mejor sobre las propiedades que poseen [17].Los 


\section{8}

sistemas constructivos en los que se enfoca este proyecto de investigación son los sistemas constructivos en tierra, más específicamente, el adobe.

Porconsiguiente, otra investigación de Moscoso en el año 2016. Se denomina El adobe, sus características y el confort térmico en cual se describe el proceso de elaboración del adobe en donde se fundamenta y da como objeto que el adobe se elabora con una mezcla de arcilla, arena y agua, aunque generalmente se le adiciona un elemento como su metodología para que ayude a los esfuerzos de corte como puede ser la paja; dicha mezcla es vertida en moldes de madera en forma de bloques y secada al sol. Evidentemente no cualquier tierra es la óptima para la elaboración de adobes, por lo que es necesario hallar, en las cercanías, una tierra que posea los porcentajes adecuados de arcilla (15\%), arena $(70 \%)$, por lo que para ello se puede realizar pruebas empíricas como la prueba del rollo".

También encontramos otra investigación realizada por Morales Domínguez en México en el año 2007. Esta investigación se denomina Mejoramiento de las propiedades mecánicas del adobe compactado en la que nos explican las propiedades que posee el adobe compactado, dice: El adobe compactado surge como una alternativa para la utilización del suelo como material de construcción, ya que cuenta con mejores características mecánicas, en el mejoramiento del proceso de fabricación y propiedades estructurales durante por el proceso de compactación pero requiere de estudios y experimentaciones para mejorar su calidad [18].

El adobe compactado tiene mejores propiedades a las del adobe sin compactar, es por eso que se pretende mejorar las características mecánicas del adobe compactado respecto a estudios previos desarrollados en el estado de Oaxaca [19]. Por último, otra investigación hallada fue hecha por Minke en Perú en el 2018, se denomina Confort térmico en una habitación de adobe con sistema de almacenamiento de calor en los andes del Perú y dice lo siguiente: El adobe es un material termorregulador, que mantiene los ambientes más abrigados en climas fríos y más frescos en climas cálidos; al ser un material proveniente del suelo, los adobes son sujetos a la dinámica de la temperatura del suelo, por eso presenta esta característica. Las construcciones de adobe tienen mayor sostenibilidad por poseer cualidades térmicas, propiedades acústicas e impacto ambiental, siendo esto beneficioso con el medio ambiente [20].La construcción sostenible de una casa se logra utilizando recursos naturales como el barro, madera y yeso, para conservar la energía y lograr el confort térmico al interior de la habitación. [21].

Materiales y métodos

En esta investigación se da un manejo de enfoque cualitativo donde se recolecta la información a partir de la observación y análisis de los datos obtenidos por medio de recopilación de diferentes fuentes bibliográficas (artículos científicos, libros, investigaciones). El diseño de investigación es de tipo descriptivo, en el que se manejo un proceso de búsqueda de información a través de fuentes variadas, sobre el adobe, sus características, ventajas y desventajas, que tan frecuente es el uso de este material y la construcción de infraestructura que requiera el uso del adobe, para analizar esta información e incentivar a la investigación de procesos que hagan más efectivo $y$ concurrido el uso del adobe como principal material de construcción.

En esta investigación la variable es la importancia del adobe en las construcciones, las ventajas y desventajas que trae consigo, la frecuencia con que es utilizado, la cantidad de edificaciones que han sido construidas a 
base de adobe, la tendencia de este material en Colombia, que regiones de Colombia es más utilizado el adobe como principal material de construcción, el concepto del adobe ante la sociedad, la implementación de nuevos proyectos que requieran el uso del adobe.

El instrumento de recolección utilizado fue el análisis documental, que consiste en la recopilación de los distintos documentos que tratan el adobe, para analizarlos y dar posibles soluciones a la problemática ambiental, recursos financieros, obtención de materiales, tiempo y costos de obra, entre otros. Y por último, las técnicas utilizadas para el procesamiento y análisis de datos, fueron las técnicas lógicas que son el análisis de toda la información obtenida por las distintas fuentes acerca del tema a tratar, en este caso el adobe y los temas relacionados con el uso y construcción referentes al adobe como tendencia en las construcciones.

\section{Resultados}

El adobe compactado es una mejor opción ya que presenta mejores características mecánicas a través del proceso de fabricación $y$ de las propiedades estructurales, siempre y cuando este se encuentre en constante estudio para el mejoramiento de la calidad.

Es por esto, que se presentan distintos estudios para cumplir con el objetivo, entre estos se encuentra el estudio de mezclas para el mejoramiento de adobe, que consiste en la elaboración del adobe compactado a través de la disminución de variables como la temperatura, humedad, grado de compacidad, mezclado y manejo de los especímenes, para esto se llevó a cabo el diseño de mezclas y se evaluó de distintas maneras para la elaboración del adobe, llevando a la práctica estos procedimientos a través de experimentos; otro estudio es el de mezclas cemento-suelo para el junteo del adobe con los estudios realizados en el diseño de mezclas, fueron analizados y llevados a distintos procesos para el mejoramiento del adobe, examinando cada una de las propiedad y exponiéndose a diferentes factores.

\section{Discusión}

La tierra es un material utilizado alrededor del mundo debido a su fácil acceso y desarrollado a través de varias técnicas dependiendo del lugar.

En México, Perú y Colombia un porcentaje considerable de las viviendas totales son construidas en tierra sobre todo en la región andina, a través de la utilización del adobe. En primera instancia los seres humanos empezaron a utilizar técnicas para dar mayor facilidad a sus labores y encontraron una alternativa en la tierra como material de construcción, cada técnica varía dependiendo de las condiciones a las que se enfrentan, entre estas técnicas se encuentra el adobe como material constructivo, elaborado a través de la mezcla de arcilla, arena y agua $y$ en varios casos adicionando elementos como paja, moldeadas en forma de bloques y secadas al sol.

No cualquiertierra esóptima para la elaboración de los adobes, por lo que previamente se realiza un estudio para saber el porcentaje de cada uno de los elementos utilizados y allí seleccionar las tierras necesarias para la fabricación de estos bloques, su tamaño varía dependiendo de las condiciones, el lugar y la estructura de la casa, siempre y cuando está brinde estabilidad. Este material es muy sensible a la humedad por lo que vendría siendo una desventaja, pero por otra parte es térmico, en climas cálidos da frescura a la vivienda y en climas fríos lo contrario, también es un material aislante, además de estas ventajas, estas construcciones no son complejas por lo que es fácil la obtención de los materiales. 


\section{0}

En Colombia el surgimiento de culturas Conclusiones indígenas como la muisca, ocupó la mayor parte de la región andina, gran parte de su cultura constructiva fue elaborada con materiales perecederos, implantando las técnicas y sistemas como el adobe dónde se observan hiladas de forma horizontal y vertical hasta la formación de muros. La arquitectura en tierra de nuestro país ha tenido gran relevancia por el significado y el valor histórico, como centros y monumentos realizados a partir de esta técnica; en la actualidad ha ido evolucionando de acuerdo a las condiciones locales. En los departamentos de Boyacá y Cundinamarca, en los centros urbanos se encuentran muros de adobe y tapia, formando un legado arquitectónico ya que hacen parte del espacio público y el paisaje del lugar.

En los altiplanos cundiboyacense, payanense y nariñense esta técnica ha tenido un desarrollo histórico y social ya que es una tradición en la construcción, en estas zonas aún se conservan edificaciones en adobe y se estableció un vínculo entre producción y viviendas, relacionadas con su bajo costo, sostenibilidad y que la materia prima está presente en el lugar haciendo de la producción algo artesanal incentivando el desarrollo sostenible a base de esta técnica.

El adobe presenta buena confección y consistencia, ya que ha perdurado en el tiempo, dando estabilidad, pero presenta un pequeño deterioro por obras de infraestructura aledañas a estas. La arquitectura del adobe en estas zonas señaladas como alta y mediana amenaza sísmica, promueve a desarrollar una norma sismo resistente con respecto a la reglamentación para las construcciones con adobe. La utilización de la tierra en la construcción ha tenido gran importancia, el municipio de Barichara es un ejemplo de ello, ya que su arquitectura recalca la importancia de este lugar dándole sentido a través de sus construcciones.

La mayoría de los materiales utilizados en la actualidad afectan al medio ambiente, pero al utilizar materiales como el adobe que es proveniente de la tierra se disminuye ese impacto medioambiental, sin embargo su uso ha disminuido por el mal comportamiento ante sismos e inundaciones.

El cemento, el acero y demás materiales provenientes de procesos industriales responden de manera eficaz en la solución de los problemas habitacionales en contextos urbanos pero su margen de efectividad se restringe cuando se trata del sector rural, de los sitios patrimoniales o de las poblaciones apartadas de los centros de producción. Es en estos lugares en donde la construcción con tierra representa una verdadera alternativa de desarrollo sostenible, y para que esta alternativa se dé, son necesarios el interés de la academia, la validación de la investigación que conlleve la legalización y la voluntad del Estado representada en políticas públicas que permitan el desarrollo, siendo el adobe material importante en la implementación de los proyectos.

\section{Referencias}

[1].J.C. Rivera Torres, "El adobe y otros materiales de sistemas constructivos en tierra cruda: caracterización con fines estructurales", Apuntes, vol.25, n.2, pp.164-181, 2012

[2].A. Holguino Huarza, L.E. Olivera Marocho y K.U. Escobar Copa, "Confort térmico en una habitación de adobe con sistema de almacenamiento de calor en los andes del Perú.", Revista de Investigaciones Altoandinas, vol. 20, n.3, pp.289-300, 2018

[3] A.N. Leyva, "Adobe", 2014. [En línea]. Disponible en: https://es.scribd.com/ document/266252284/Adobe 
[4] V. Morales-Domínguez, M. Ortiz-Guzmán y R. Alavéz-Ramírez, "Mejoramiento de las propiedades mecánicas del adobecompactado", Naturaleza y Desarrollo, vol. 5, no. 1, 2007

[5] S. Rivero Bolaños, "El uso masivo de la tierra como material de construcción en Colombia.", APUNTES, vol. 20, no. 2, pp. 354-363, 2007

[6] R. Rotondaro, "Arquitectura de tierra contemporánea: tendencias y desafíos", APUNTES, vol. 20, no. 2, pp. 342-353, 2007

[7].C.E. Sánchez Gama, "La arquitectura de tierra en Colombia, procesos y culturas constructivas", APUNTES, vol. 20, no.2, pp. 242-255, 2007

[8] G.M. Viñuales, “Tecnología y construcción con tierra”, APUNTES, vol. 20, no. 2, pp. 220-231, 2007

[9] J.V. Quintero Garzón "Centro de Convenciones y Emprendimiento en Villa de Leyva, Boyaca, Colombia", trabajo de grado, Universidad Católica de Colombia, 2019

[10] Y. Contreras-Valero, Y. Cardona-Rodríguez, y D. A. Torres-Sánchez, "Estudio térmico e infrarrojo de cinco tipos de madera comercializadas en Norte de sSntander, para su posible uso como fuente de energía", Respuestas, vol. 22, no 2, pp. 36-47, jul. 2017

[11] A. Sarabia-Guarin, J. Sánchez-Molina, y J. C. Leyva-Díaz, "Uso de nutrientes tecnológicos como materia prima en la fabricación de materiales de construcción en el paradigma de la economía circular", Respuestas, vol. 22, no. 1, pp. 6 - 16, ene. 2017

[12] G.M. Viñuales, “Tecnología y construcción con tierra", APUNTES vol. 20, núm. 2 220-231, 2007

[13] C.E. Sánchez Gama, "La arquitectura de tierra en Colombia, procesos y culturas constructivas", Apuntes, vol. 20, no.2, 2007
[14] J.E. Gama-Castro, et al., "Arquitectura de tierra: el adobe como material de construcción en la época prehispánica", Bol. Soc. Geol. Mex, vol.64, no.2, 2012

[15] C. Uribe Vélez, "Materiales y prácticas de construcción sostenible", Universidad EAFIT, Medellín - Antioquia, 2012

[16] A. Castro, "Economía, salud, desarrollo humano e innovación en el desarrollo sustentable", Conocimiento global, vol. 3, no. 1, pp. 1-13, 2018

[17] E. Alzamora, "El poder del conocimiento y de la información como generador de valor en las organizaciones", Conocimiento global, vol. 3, no. 1, pp. 14-29, 2018

[18] A. Caballero Tovío, “Grado de articulación de las estrategias consignadas en el Plan Nacional de Desarrollo 2014-2018 con la situación del sector industrial colombiano", Enfoque Disciplinario, vol. 1, no. 1, pp. 14-20, 2016

[19] O. Bernal Payares, "Evolución del proceso administrativo desde los orígenes de la revolución industrial y su fundamento como una función del administrador", Enfoque Disciplinario, vol. 2, no. 1, pp. 1-14, 2017 